引用格式:邓荣荣, 张韩祥. 中国城市数字金融发展对碳排放绩效的影响及机理[J]. 资源科学, 2021, 43(11): 2316-2330. [Deng R R, Zhang A X. The impact of urban digital finance development on carbon emission performance in China and mechanism[J]. Resources Science, 2021, 43(11): 2316-2330. ] DOI: 10.18402/resci.2021.11.14

\title{
中国城市数字金融发展对碳排放绩效的 影响及机理
}

\author{
邓荣荣, 张朝祥
}

(南华大学经济管理与法学学院, 衡阳 421001)

\begin{abstract}
摘 要:在数字经济时代, 实现碳达峰、碳中和, 是一场广泛而深刻的经济社会系统性变革,需要利用数字金融 形成节能减排的长效机制。本文在阐述数字金融影响城市碳排放绩效理论的基础上,运用2012-2018 年中国 285 个城市作为研究样本, 运用面板回归模型、工具变量法、中介效应模型等方法实证分析了数字金融发展对碳排放绩 效的影响方向及其作用机理。结果表明: (1) 数字金融发展显著降低了碳排放强度和提高了碳排放效率,在整体上 改善了碳排放绩效,并且数字金融覆盖广度、使用深度和数字化程度的提升均改善了碳排放绩效。(2)数字金融发 展通过经济增长效应、产业结构效应和技术创新效应降低了碳排放强度, 通过经济增长效应和技术创新效应提升 了碳排放效率。总体来看, 数字金融发展通过这 3 种效应改善了碳排放绩效。(3)数字金融发展更有利于低经济发 展水平和低金融发展水平城市碳排放强度的降低; 而在碳排放效率方面, 数字金融发展更有利于高经济发展水平 和高金融发展水平城市碳排放效率的提升。未来中国政府应加快发展数字金融,促进金融服务与现代数字技术结 合, 通过优化金融资源配置为碳达峰、碳中和目标提供支撑。
\end{abstract}

关键词: 数字金融;碳排放强度; 碳排放效率; 中介机制;异质性影响; 中国

DOI :10.18402/resci.2021.11.14

\section{1 引言}

改革开放以来中国经济保持着高速增长, 但长 期以来以要素驱动为特征的粗放型发展方式引致 了大量的能源消耗和碳排放, 不利于经济社会的可 持续发展。为控制温室气体排放, 中国政府在 2016 年《巴黎协定》中提出了自主减排承诺: 到 2030 年碳 排放达峰, 碳排放强度与 2005 年相比降低 $60 \%$ $65 \%$ 。推进低碳经济发展成为 “新常态”阶段中的一 项重要且严峻的任务。与此同时,依托于互联网、 大数据、云计算等数字技术, 中国的数字金融正经 历着快速发展, 大幅提高了金融资源的可得性和可 利用性。数字金融已经渗透到生产生活中的方方 面面, 对企业生产、居民生活都带来新变革。那么
数字金融发展是否对碳排放绩效产生了影响? 若 产生了影响, 这种影响是通过何种机制实现的? 上 述问题的回答对于丰富数字金融理论体系、促进碳 减排具有理论与现实意义。

已有的有关数字金融的研究中,部分文献关注 了数字金融对环境的影响, 如贺茂斌等 ${ }^{[1]}$ 以产业碳 排放作为中介变量研究了数字金融的生产率效应, 发现数字普惠金融可通过降低区域碳排放提升全 要素生产率; 陈矜等 ${ }^{[2]}$ 研究发现,数字金融可通过缓 解收人不均衡和促进产业结构绿色化减少环境污 染, 但也会通过促进收人增长加剧环境污染。许钊 等 ${ }^{[3]}$ 研究了数字金融的污染减排效应及其作用机 制, 发现数字金融通过创业创新效应和产业升级效

收稿日期: 2021-03-30,修订日期: 2021-06-04

基金项目:湖南省社会科学基金项目(20YBA226);湖南省研究生科研创新项目(CX20210949)。

作者简介: 邓荣荣, 女, 湖南衡阳人,博士, 副教授, 硕士生导师, 研究方向为低碳经济。E-mail: 2419047387@qq.com

通讯作者: 张鼿祥,男,山东临沂人,硕士研究生,研究方向为低碳经济。E-mail: 775126196@qq.com 
关系,外商投资规模对中国碳排放强度的影响存在

数字金融是传统金融与现代科技融合的结果， 仍具备传统金融的基本特征, 因此有关传统金融对 碳排放影响的研究可以为本文提供借鉴。有关金 融发展与碳排放关系的研究主要沿如下脉络展开: (1)金融发展对碳排放的影响效应研究。主要分为 两种观点:第一种观点认为金融发展能够显著抑制 能源消耗,有利于碳减排目标的实现, 如 Artur 等 ${ }^{[4]}$ 最早研究了金融发展与环境质量的关系, 认为金融 发展能够抑制环境质量退化, 金融自由化是减少二 氧化碳的重要因素。Ouyang 等 ${ }^{[5]}$ 采用信贷、保险业 收人、股票市场价值等指标构造了金融发展指数, 研究发现金融发展可以显著降低各地区能源消耗, 进而降低碳排放; 贺俊等 ${ }^{[6]}$ 研究发现金融机构信贷 规模、金融市场融资规模与环境污染呈负相关, 且 技术效应起到了中介效应的作用。第二种观点认 为金融发展促进了碳排放的增加, 持这一观点的学 者认为技术创新、外商直接投资等在金融发展与环 境污染关系之间存在中介效应 ${ }^{[7]}$, 金融市场化水平 的提升通过引人研发投资和外商直接投资促进了 技术水平的提升, 经济社会在通过技术进步效应快 速增长的同时对能源消耗产生更大规模的需求, 通 过回弹效应引致能源消耗和碳排放的增加 ${ }^{[8]}$, 此外, 金融发展在促进外商直接投资增加的同时也可能 导致“污染避难所”效应,对东道国的碳减排产生负 面影响。(2)金融发展对碳排放影响的异质性分 析。不少研究指出, 金融发展对碳排放的影响存在 区域异质性, 如邵汉华等 ${ }^{[9]}$ 研究发现金融发展总体 上有利于降低碳排放, 但在人均 GDP 水平、工业比 重、煤炭消费比重和贸易开放程度等较高的省份, 金融发展的碳减排效应相对较低; 彭智敏等 ${ }^{\left[{ }^{[0]}\right.}$ 以长 江经济带为实证样本, 研究发现东部地区金融规模 与碳排放存在倒 $U$ 型关系, 而中部和西部地区金融 规模促进了碳排放的增加; (3)金融发展对碳排放或 碳排放强度的影响机制研究。现有的有关金融发 展对环境污染影响机制的研究基本沿用 Grossman 等 ${ }^{[1]}$ 关于经济活动环境效应的 3 类分解, 即规模效

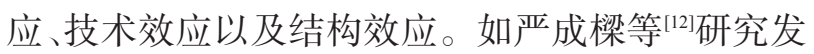
现信贷规模对中国碳排放强度的影响存在倒 $U$ 型 $\mathrm{U}$ 型关系, 其影响方向取决于金融发展所引起的规 模效应和技术效应在不同阶段的权衡; 熊灵等 ${ }^{[13]}$ 研 究发现无论是从人均碳排放角度还是碳排放强度 角度来看,金融发展对中国省区碳排放都起到负面 作用,其主要原因在于金融发展的财富效应和规模 效应大于技术效应和结构效应; 陈碧琼等 ${ }^{[14]}$ 研究发 现中国金融规模扩大对碳排放变化所产生的规模 效应超过了技术效应和结构效应,促进了碳排放量 增加; 胡宗义等 ${ }^{[15]}$ 研究发现, 金融发展对环境污染的 影响机制存在门槛特征, 金融发展水平较低时, 金 融发展对污染排放影响规模效应大于技术效应,具 有显著促进作用, 当金融发展水平较高时,规模效 应小于技术效应, 金融发展对污染排放具有显著抑 制作用。

综上所述, 现有文献从不同的角度研究了传统 金融发展对碳排放的影响。当前以大数据、人工智 能、区块链为代表的数字技术正持续推动中国货币 金融数字化创新实践，在支付、信贷、保险、投资等 领域的使用深度不断增加, 改变着传统金融的服务 形态与模式,数字金融在经济金融领域的渗透也可 能通过一定的作用机制影响碳排放。鉴于此,本文 聚焦数字金融发展对城市碳排放绩效的影响效应 及作用机制, 基于 285 个地级及以上城市 20122018 年的面板数据, 分析数字金融发展对碳排放强 度和碳排放效率的影响, 并深人探讨了数字金融影 响碳排放绩效的中介效应和异质性。本文的研究 从数字金融的角度拓展了金融发展与碳排放绩效 关系的研究,为更好地发挥数字金融的碳减排效应 提供稳健的实证依据和决策参考。

\section{2 理论分析}

\section{1 数字金融发展对碳排放绩效的直接影响}

数字金融是传统金融与互联网、大数据、云计 算数字技术结合形成的金融服务形态, 是对传统金 融服务的进一步发展。数字支付、网络信贷等数字 金融服务本身就是环境友好型的服务形态，例如， 金融机构可通过线上平台直接向企业提供信贷产 品, 避免了企业在线下往返金融机构造成的碳排放 的增加。 
数字金融发展对碳排放绩效的影响还包括以 下几个方面: (1)碳交易被认为是通过市场规制工具 影响碳排放、应对气候变化的重要手段,数字金融 可以使碳金融产品更加普惠化和大众化, 推进碳交 易市场的扩大, 对没有惩罚或没有补偿的碳排放行 为通过产权定价、市场交易的方式进行平衡, 最终 将碳排放的外部性内部化, 有利于碳减排; (2)金融 机构可以通过数字化平台对企业进行遴选, 淘汰高 能耗高污染的企业,引导金融资源向环境友好型企 业流动, 建立基于数字化平台的绿色金融资源配置 与管理生态系统 ${ }^{[1]}$ 。(3)数字金融平台可以对企业信 息进行搜寻、分类和计算,为政府提供海量的环境 信息,降低政府的信息搜寻成本,使其能够获取准 确的环境信息, 实现科学决策和精准规制, 对企业 进行 “奖优惩劣”, 促进城市碳减排。因此本文提出 以下假设：

\section{$\mathrm{H} 1$ : 数字金融发展改善了碳排放绩效。}

\section{2 数字金融发展对碳排放绩效的影响机制}

数字金融是对传统金融服务的延伸和补充。

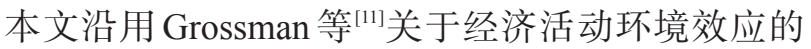
分解思路探讨数字金融对碳排放绩效影响的作用 机制: (1)数字金融会通过提升企业绩效、增加消费 者需求促进经济增长, 激活经济增长潜力 ${ }^{[17]}$, 从而对 碳排放绩效产生影响; (2)数字金融能通过融资激励 增强企业技术创新的动力, 并通过融资支持加强企 业技术创新能力 ${ }^{[18]}$, 产生的技术创新效应将影响城 市碳排放绩效的变化; (3)数字金融会通过增加金融 服务业产值、提升要素配置效率促进中国产业结构 的高级化和合理化 ${ }^{[19]}$, 从而对碳排放绩效产生影 响。综上, 本文认为数字金融将会通过经济增长效 应、技术创新效应和产业结构优化效应影响碳排放 绩效,具体的影响方向及内涵分析如下。

\subsection{1 数字金融通过促进经济增长改善碳排放绩效}

数字金融对经济增长的影响体现在金融资源 的收集与供给水平的提升。首先, 就金融资源收集 来说, 金融机构能够利用大数据、云计算、分布式记 账等前沿数字技术以较低的交易成本收拢游离于 正规金融机构外的金融资源, 将其转化为有效供 给, 为企业提供充足的信贷支持, 同时数字技术可
将线下金融服务交易转移到线上平台,简化了业务 流程,便利了各区域之间的金融服务交易,促进金 融资源的优化配置。其次, 就金融资源供给来说, 数字金融具有普惠性特征, 可以降低获得金融服务 的成本和门槛, 催生出“长尾市场”, 提高弱势群体 金融资源的可利用性,改善民营企业和小微企业融 资贵、融资难的状况, 增加企业生产绩效, 同时数字 技术的应用可以帮助金融机构对企业信息进行搜 索、分析、决策,筛选出具有发展潜力的民营和小微 企业, 为其提供金融支持, 促进经济增长。广泛的 研究表明经济增长与人均碳排放存在倒 “U”型关

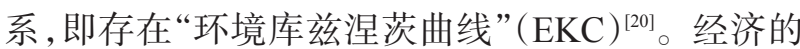
高速增长需要消耗大量的能源资源并产生大量的 碳排放, 碳排放绩效随之恶化; 当经济发展到一定 的水平时,居民和政府将会更加重视环境质量,追 求可持续发展,政府将增加污染治理投资,促进碳 减排,现阶段的研究结果表明未来一段时间内经济 增长将有利于降低碳排放 ${ }^{[21]}$ 。

\subsection{2 数字金融通过激发技术创新改善碳排放绩效}

技术创新是降低污染排放的重要因素 ${ }^{[22,23]}$ 。企 业的创新行为具有的经济增长效应、资源与环境效 应、结构优化效应等 ${ }^{[24]}$, 是促进碳排放绩效提升的重 要手段。就企业的绿色技术研发而言,其具有投人 沉没性、过程不可逆、成果不确定、融资成本高等特 征, 研发成果的产出和转化具有较长的时间滞后, 需要充足的金融资源予以支持。而传统的金融服 务体系往往通过判断企业的可抵押资产、后续的盈 利能力、现期市场规模等条件䇻选授信客户, 具有 短视性, 导致真正具有市场潜力的、高研发强度的 企业难以获得融资支持, 抑制了绿色技术创新。数 字金融的发展则可以有效缓解金融资源错配的情 况,为企业的技术研发提高融资支持。数字金融对 企业绿色创新的促进作用体现在: (1)数字金融具有 普惠性特征,可以为中小企业的技术研发和创新活 动提供融资支持。(2)内嵌于数字金融体系的大数 据、云计算等数字技术,可以对用户消费信息进行 搜寻、分析、决策,帮助实体企业对创新方向、创新 潜力、创新路径等进行判断, 促使企业创新由经验 驱动向数据驱动转变, 提高技术研发的成功率和研 
发成果的转化能力。企业作为产业经济的细胞, 其 绿色技术创新将最终促进宏观绿色技术的进步, 促 进碳排放绩效的提升。

\subsection{3 数字金融通过优化产业结构改善碳排放绩效}

数字金融可以促进产业结构的转型升级,主要 体现在产业结构高级化和产业结构合理化两方 面。就产业结构高级化来看: 首先, 数字金融作为 服务业的一部分, 其发展促进了自身产值的增加， 提高了服务业产值的占比。其次,数字金融发展可 以显著缓解要素错配, 降低要素重置成本,引导生 产要素向效率更高的部门转移, 促进产业结构升 级, 第三产业为经济贡献高、能耗低、排放少的绿色 产业, 因此产业结构高级化将对碳排放绩效产生有 利影响 ${ }^{[25]}$ 。就产业结构合理化来看: 数字金融促进 了金融资源的有效配置, 依托数字化技术, 有效弱 化了信息不对称问题,降低了信任风险, 提高了金 融资源配置效率, 促进产业结构合理化。其次, 数 字金融对中小企业进行融资支持, 催生出新的就业 岗位, 并为弱势群体提供人力资本投资, 进而产生 劳动力蓄水池效应、人力资本效应, 弱化劳动力错 配对资源配置效率的消极影响 ${ }^{[19]}$, 促进产业结构合 理化。产业结构合理化将提高能源等各类资源要 素的利用效率 ${ }^{[26]}$, 推进经济绿色发展, 对碳排放绩效 产生有利影响。

基于以上分析, 本文提出以下假设:

$\mathrm{H} 2$ : 数字金融通过经济增长效应、技术创新效 应和产业结构效应 3 种中介渠道改善碳排放绩效。

\section{3 研究方法与数据来源}

\section{1 研究方法}

\subsection{1 模型设定}

根据以上的理论分析可知数字金融发展会对 城市碳排放绩效产生影响, 为验证数字金融发展对 城市碳排放绩效的影响, 参考钱海章等 ${ }^{[2]}$ 的研究, 设 定线性回归模型：

$$
Y_{i t}=\beta_{0}+\beta_{1} D f_{i t}+\beta_{2} C_{i t}+u_{i}+\mu_{i t}
$$

式中: $i$ 表示城市; $t$ 表示年份; $Y$ 表示碳排放强度 $(C i)$ 或碳排放效率 $(C e) ; D f$ 表示数字金融指数; $C$ 表示 一系列影响碳排放绩效的控制变量; $u$ 表示不随时 间变化的个体效应; $u$ 表示随机扰动项; $\beta_{0}$ 表示常
数项; $\beta_{1} 、 \beta_{2}$ 表示待估计系数。

式 (1) 仅关注了数字金融发展对碳排放绩效影 响的总效应,其具体的影响机制如何? 需要对中介 效应进行分析。根据前面的理论分析可知,数字经 济发展主要是通过经济增长效应、产业结构效应和 技术创新效应 3 种途径对碳排放绩效产生影响。本 文试图对上述中介效应进行实证检验, 参考温忠麟 等 ${ }^{[28]}$ 的研究,建立以下中介效应模型:

$$
\begin{aligned}
& M_{i t}=\beta_{0}+\beta_{1} D f_{i t}+\beta_{2} C_{i t}+u_{i}+\mu_{i t} \\
& Y_{i t}=\alpha_{0}+\alpha_{1} D f_{i t}+\alpha_{2} M_{i t}+\alpha_{3} C_{i t}+u_{i}+\mu_{i t}
\end{aligned}
$$

式中: $M$ 表示中介变量,包括经济发展水平 $(R g d p) 、$ 产业结构 $(I n s)$ 和技术水平 $(T e c) 。 \alpha_{0}$ 表示常数项; $\alpha_{1} 、 \alpha_{2} 、 \alpha_{3}$ 表示待估计系数。系数 $\beta_{1}$ 与 $\alpha_{1}$ 的乘积 即为本文所关注的中介效应的大小。

\subsection{2 变量定义}

\section{(1) 被解释变量}

本文的被解释变量为碳排放强度 $(C i)$ 和碳排放 效率 $(C e)$ 。前者通过计算城市碳排放与地区实际 生产总值的比值获得 ${ }^{[29]}$, 后者参考马大来等 ${ }^{[30]}$ 、王少 剑等 ${ }^{[31]}$ 的研究, 以劳动力、资本、能源为投人要素, 以 碳排放为非期望产出, 以地区生产总值为期望产 出, 采用包含非期望产出 SBM-Undesirable 模型来 计算。碳排放强度越小、碳排放效率越高, 表明碳 排放绩效越好。

关于碳排放的计算, 由于城市尺度并没有详细 的能源消费和分类数据, 本文借鉴 $\mathrm{Chen}$ 等 ${ }^{[32]}$ 的研 究, 采用 NPP-VIIRS 夜间灯光数据降尺度反向推演 中国城市碳排放。基于 NPP-VIIRS 夜间灯光数据 进行碳排放的模拟测度近年来在经济学研究领域 应用非常广泛 ${ }^{333,34]}$, 基本逻辑为: 夜间灯光亮度越 高,城市的夜间经济活动越活跃,该城市的经济发 展水平越高, 能源消费也会越多 ${ }^{[29]}$ 。参考吴健生 等 ${ }^{[35]}$ 的做法, 考虑到降尺度模型反演的精度问题, 采 取不含截距的线性模型对碳排放进行拟合, 各省碳 排放估计值和统计值进行相关性检验结果如图 1 所 示, 可以看到碳排放估计量与统计量存在正线性相 关性, 拟合优度 $R^{2}$ 达到了 0.8668 左右, 拟合优度较 高, 说明碳排放估计量与统计量之间存在较强的相 


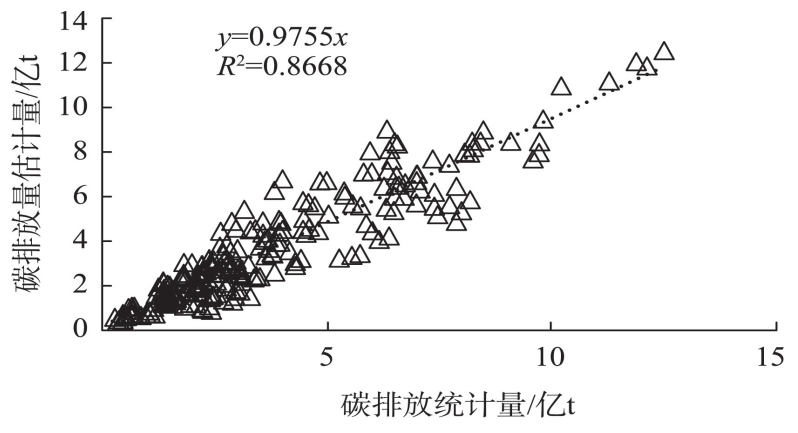

图 1 省份碳排放估计量与碳排放统计量的相关性

Figure 1 Correlation between provincial carbon emission estimates and carbon emission statistics

关性, 采用夜间灯光数据反向推演中国城市碳排放 的方法具有一定的科学性和有效性。

(2)核心解释变量

本文的核心解释变量为数字金融指数 $(D f)$, 采 用数字普惠金融指数作为数字金融指数的代理变 量, 该数据源自于北京大学互联网金融研究中心在 2020 年发布的《数字普惠金融指数》 ${ }^{[36]}$, 该指数是在 蚂蚁金融提供的金融服务数据基础上合成的,衡量 了中国省级、城市级以及县级数字金融发展程度。 数字金融指数包括 3 个子维度, 分别是覆盖广度、使 用深度和数字化程度, 其中覆盖广度 $(B r e)$ 主要包含 支付宝账号数量、支付宝绑卡用户比例和支付宝账 号绑卡数量 3 项指标, 体现的是数字金融的覆盖率; 使用深度 $(D e p)$ 涵盖支付、信贷、保险、投资、信用以 及货币基金等服务,体现的是数字金融工具种类的 增多和可得性的提升; 数字化程度 (Dig) 涵盖移动 化、实惠化和便利化, 体现的是数字金融与数字技 术的融合程度以及普惠性程度。本文采用这 3 个子 指标作为解释变量研究数字金融不同维度对碳排 放绩效的影响。

(3) 中介变量

本文的中介变量包括: (1) 经济发展水平 $(E c o)$, 采用人均实际 $\mathrm{GDP}$ 表示。2 技术水平 $(\mathrm{Tec})$, 采用 城市专利申请量表示, 借鉴白俊红等 ${ }^{\left[{ }^{37}\right]}$ 的研究按照 创新程度的高低分别对发明专利、实用新型以及外 观设计 3 种专利赋予 $0.5 、 0.3 、 0.2$ 的权重, 加总并取 对数, 以综合衡量专利数量和专利质量。(3)产业结 构 (Ins), 采用余弦法计算产业结构升级指数, 具体 计算方法参见付凌晖 ${ }^{[38]}$ 的研究。

\section{(4)控制变量}

本文参考以往的文献 ${ }^{[39-41]}$, 控制了以下可能影 响碳排放绩效的变量: (1)人口密度 $(P i)$, 采用平方千 米的人口数表示。(2)财政支出 $(G o v)$, 采用财政支 出与 GDP 的比值表示。(3)外商直接投资 $(F d i)$, 采 用实际利用外资与 GDP 的比值表示。(4)环境规制 $(E r)$, 借鉴广嫦娥 ${ }^{[42]}$ 的做法, 采用市辖区城区绿化覆 盖率作为环境规制的代理变量,一般绿化覆盖率越 高, 城市的环境规制强度越强。(5)金融发展水平 $(J r)$, 采用金融机构年末存贷款总额与 GDP 的比值 表示。(6)道路面积 $(R s)$, 采用城市道路面积与城市 区域面积比值表示。各变量的描述性统计见表 1 。

\section{2 数据来源}

为了保证样本数据的连续性和可得性,结合行 政区划调整和数据缺失的情况,本文选取了 285 个 地级及以上城市 (因数据缺失, 不包括港澳台地区) 作为实证研究的样本, 样本时间为 2012-2018 年。 本文的夜间灯光灰度值数据来源于全球夜间灯光 数据库 (Global Night-time Light Database, GNLD) 中国各城市灯光数据(校正后), 该数据是基于 NPPVIIRS 卫星原始夜间灯光数据经过提取、校正处理 获得的。NPP-VIIRS 原始夜间灯光数据源自美国国 家海洋和大气管理局 (National Oceanic and Atmospheric Administration, NOAA）。数字普惠金融指 数源自北京大学数字金融研究中心。其他经济社 会数据均来自《中国城市统计年鉴(2013-2019)》、 各省统计年鉴以及各市的年度国民经济社会发展 统计公报。

\section{3 内生性问题}

尽管本文尽可能控制了影响碳排放绩效的变 量,但可能依然存在遗漏变量所导致的内生性问 题,并且数字金融与碳排放绩效可能存在反向因果 问题,碳排放绩效较高的地区可能更易推动数字金 融的发展。本文试图通过工具变量法缓解内生性, 参考易行健等 ${ }^{[43]}$ 的做法,构建了工具变量“Bartik instrument”, 即滞后一阶的数字普惠金融指数 $\left(D f_{i t-1}\right)$ 和数字普惠金融指数一阶差分 $\left(\Delta D f_{i t-1}\right)$ 的乘积项 $\left(D f_{i t-1} \times \triangle D f_{i t-1}\right)$ 。构建此工具变量的考虑如下: (1)全 国数字普惠金融指数来自于 285 个城市 (均值), 因 此其变化趋势不会明显受到单个城市的影响,差分 


\section{表 1 变量描述性统计}

Table 1 Descriptive statistics of variables

\begin{tabular}{|c|c|c|c|c|c|c|c|}
\hline 变量类型 & 变量 & 单位 & 样本量 & 均值 & 标准差 & 最小值 & 最大值 \\
\hline \multirow[t]{2}{*}{ 被解释变量 } & $\mathrm{Ci}$ & $t /$ 万元 & 1995 & 6.973 & 4.981 & 0.400 & 39.120 \\
\hline & $\mathrm{Ce}$ & 指数 & 1995 & 0.257 & 0.127 & 0.061 & 1.000 \\
\hline \multirow{4}{*}{ 核心解释变量 } & $D f$ & 指数 & 1995 & 170.400 & 51.060 & 55.520 & 303.000 \\
\hline & Bre & 指数 & 1995 & 159.500 & 49.700 & 36.110 & 290.300 \\
\hline & Dep & 指数 & 1995 & 167.700 & 57.130 & 27.830 & 325.700 \\
\hline & Dig & 指数 & 1995 & 211.300 & 64.260 & 51.520 & 581.200 \\
\hline \multirow[t]{3}{*}{ 中介变量 } & $R g d p$ & 万元/人 & 1995 & 5.444 & 5.152 & 0.618 & 55.680 \\
\hline & $\mathrm{Tec}$ & 对数 & 1995 & 6.237 & 1.737 & 0.001 & 11.37 \\
\hline & Ins & 指数 & 1995 & 6.510 & 0.348 & 5.517 & 7.614 \\
\hline \multirow[t]{6}{*}{ 控制变量 } & $P i$ & 百人 $/ \mathrm{km}^{2}$ & 1995 & 4.339 & 3.399 & 0.051 & 26.480 \\
\hline & $\operatorname{Reg}$ & $\%$ & 1995 & 39.250 & 7.327 & 0.590 & 95.250 \\
\hline & $F i$ & $\%$ & 1995 & 16.300 & 1.132 & 13.720 & 20.270 \\
\hline & $F d i$ & $\%$ & 1995 & 11.720 & 10.500 & 0.000 & 73.620 \\
\hline & Gov & $\%$ & 1995 & 19.700 & 13.050 & 4.388 & 234.900 \\
\hline & $R s$ & $\%$ & 1995 & 0.267 & 0.584 & 0.002 & 6.085 \\
\hline
\end{tabular}

项相对于单个城市而言可视作外生; (2)城市碳排放 绩效可能受到其他未观测冲击的影响, 但只要这种 冲击没有重要到影响全国数字普惠金融指数, 那么 这个工具变量就是有效的。

\section{4 结果与分析}

\section{1 碳排放绩效测算结果及分布特征}

采用ArcGIS10.2 软件对碳排放绩效进行可视 化分析。其中, 2012 年、2018 年碳排放强度的空间 分布如图 2 所示, 可以看到东部沿海地区、中部地区 的碳排放强度较低, 东北地区和西部地区的碳排放 强度较高, 碳排放强度呈现由东向西逐级递增的分 布特征。

图 3 显示了 2012 年、2018 年碳排放效率的空间 分布格局, 可以看到碳排放效率较高的城市主要集 中在北方的京津冀地区、呼包鄂榆地区、辽宁中南 部地区、山东半岛地区和南方的长三角地区、长江 中游地区、成渝地区以及珠三角地区, 呈现由各区 域增长极、大型城市向周围扩散、逐渐降低的分布 特征。

\section{2 数字金融发展对碳排放绩效的影响}

首先, 采用面板固定效应模型估计数字金融发 展对碳排放绩效的影响, 回归结果如表 2 第 $1 、 2$ 列 所示, 当以碳排放强度 $(C i)$ 作为被解释变量时, 数字
金融指数 $(D f)$ 的系数为负, 且在 $1 \%$ 的水平上显著， 说明数字金融发展引起了碳排放强度的降低, 降低 了单位产值的二氧化碳排放量; 当以碳排放效率 $(C e)$ 作为被解释变量时,数字金融指数 $(D f)$ 的系数 为正, 且在 $1 \%$ 的水平上显著, 说明数字金融发展促 进了碳排放效率的提升, 验证了本文的假设 1 。其 次, 采用工具变量法进行回归, 回归结果见表2第 $3 、$ 4 列, C-D Wald F 检验结果拒绝了工具变量与内生 变量相关性较弱的原假设 (Wald-F>8.96), 通过了弱 工具变量检验,说明工具变量的选取是合理的。工 具变量法的回归结果与固定效应回归结果并无显 著差异, 说明考虑了内生性后, 实证结果依然稳健。

\section{3 数字金融发展各维度对碳排放绩效的影响}

由于数字金融指数包含覆盖广度、使用深度与 数字化程度 3 个子维度, 为了进一步分析数字金融 哪些维度影响了碳排放绩效,我们分别对其进行双 固定效应模型的估计, 回归结果如表 3 所示。结果 显示, 数字金融覆盖广度和数字化程度促进了碳排 放强度的降低, 3 种指标均促进了碳排放效率的 提升。

(1)数字金融覆盖广度 $(B r e)$ 。覆盖广度的增加 表明传统金融与现代数字技术的融合使金融服务 突破原有的限制, 为广大的中小微企业提供足够的 

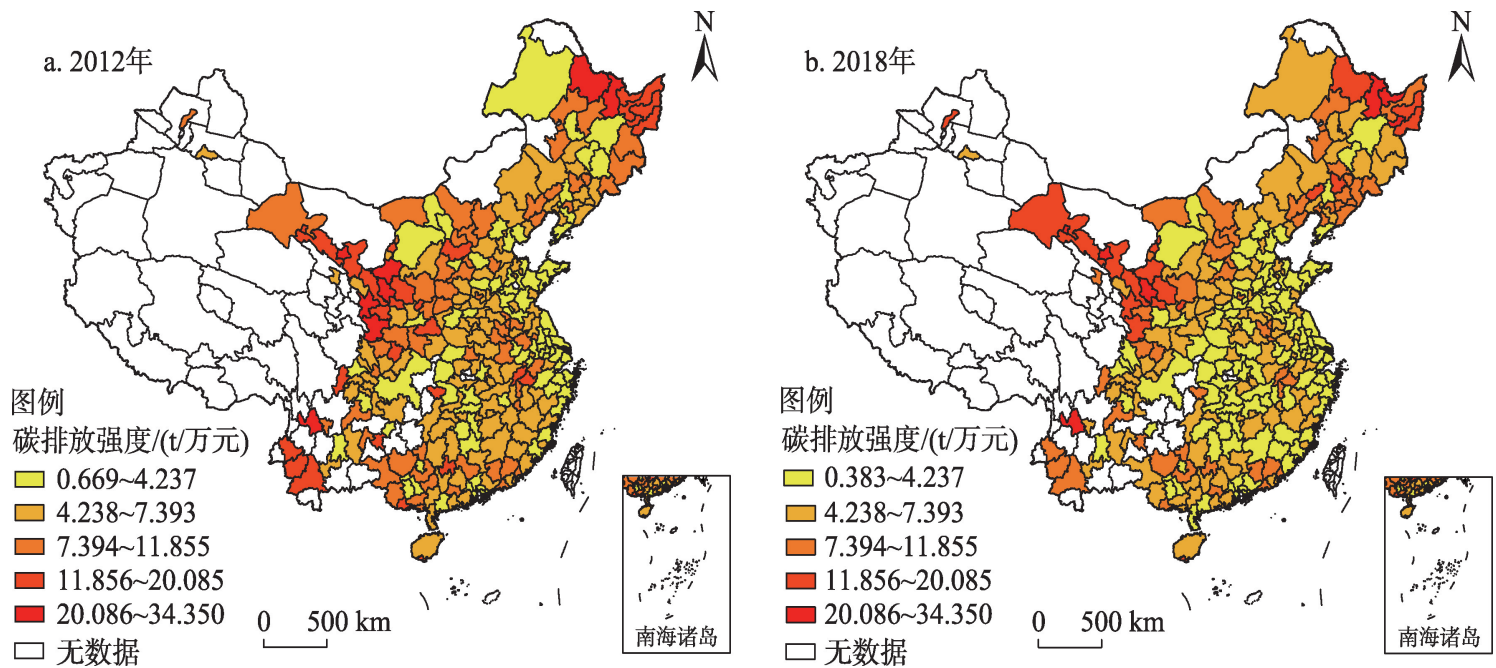

图 $22012 、 2018$ 年中国城市碳排放强度时空分布格局

Figure 2 Spatial and temporal distribution pattern of carbon emission intensity in China, 2012 and 2018 注:基于自然资源部标准地图服务网站 GS(2019)1823号标准地图制作,底图边界无修改。
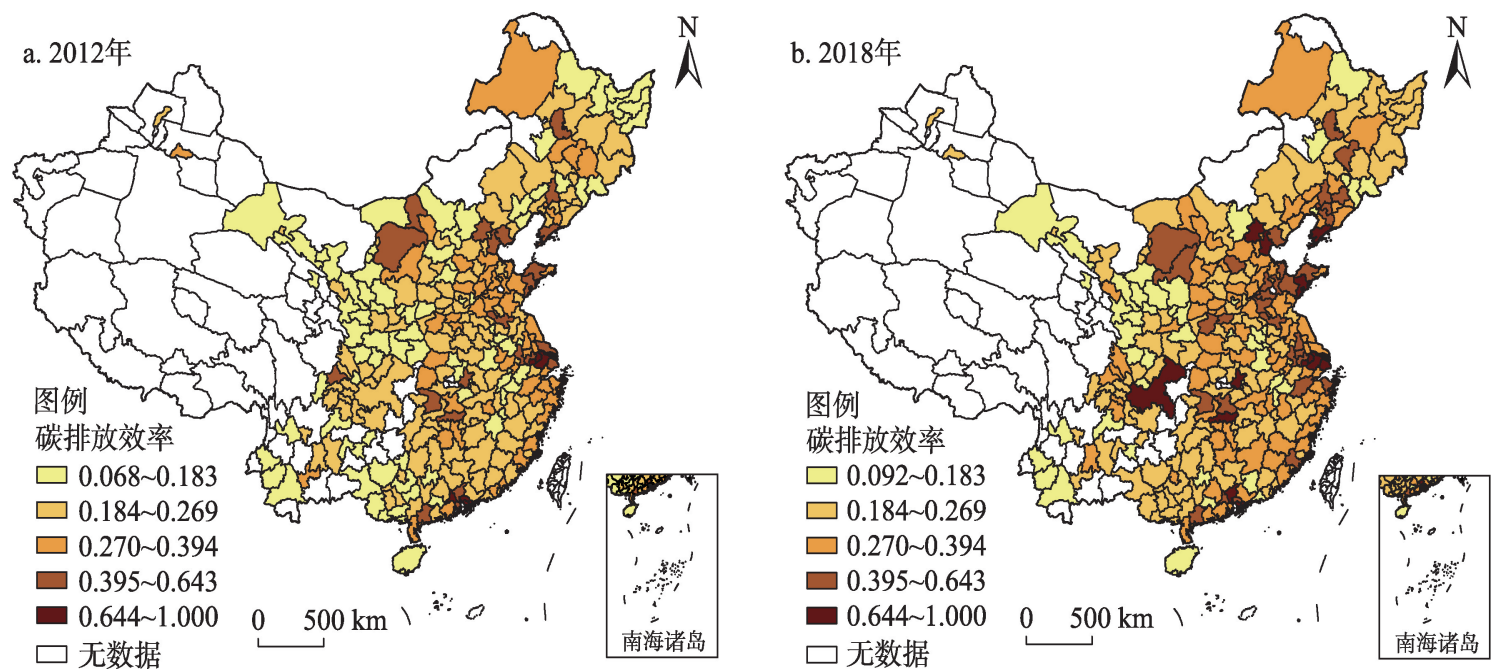

图32012、2018 年中国城市碳排放效率时空分布格局

Figure 3 Spatial and temporal distribution pattern of carbon emission efficiency in China, 2012 and 2018 注:基于自然资源部标准地图服务网站 GS(2019)1823号标准地图制作, 底图边界无修改。

数字金融服务,金融服务业产值增加, 地区产业结 构升级,有利于改善碳排放绩效。

(2)数字金融使用深度 $(D e p)$ 。使用深度指标 的提高表明支付、信贷、保险、信用、投资、货币基金 等业务的增加, 提高了金融产品的可得性, 丰富的 金融工具和产品有效满足了相应需求, 增加了企业 研发投资, 提高了企业资源利用效率, 改善了碳排 放绩效。

(3) 数字化程度 $(D i g)$ 。数字化程度的提升表
明金融与数字技术的融合水平的提升,金融机构可 通过大数据、云计算等数字化技术对企业数据进行 搜集、分析和决策,为研发能力强、有潜力的企业提 供信贷支持,通过促进企业技术创新改善碳排放绩 效。金融机构可通过线上平台直接向企业提供贷 款, 促进了金融流通效率的提升,避免企业在线下 往返金融机构造成的碳排放的增加，降低资源浪费 和效率损失,改善城市碳排放绩效。

\section{4 中介效应分析}


表 2 数字金融发展对碳排放绩效的影响

Table 2 Impact of digital finance development on carbon emission performance

\begin{tabular}{|c|c|c|c|c|}
\hline \multirow{2}{*}{ 变量 } & \multicolumn{2}{|c|}{ 固定效应回归 } & \multicolumn{2}{|c|}{ 工具变量回归 } \\
\hline & $\mathrm{Ci}$ & $\mathrm{Ce}$ & $\mathrm{Ci}$ & $\mathrm{Ce}$ \\
\hline \multirow[t]{2}{*}{$D f$} & $-0.0032 * * *$ & $0.0004 * * *$ & $-0.0433^{* * *}$ & $0.0002 * *$ \\
\hline & $(0.0012)$ & $(0.0000)$ & $(0.0076)$ & $(0.0001)$ \\
\hline \multirow[t]{2}{*}{$P i$} & -0.0281 & $-0.0105^{* *}$ & 0.0208 & $-0.0018^{*}$ \\
\hline & $(0.1021)$ & $(0.0041)$ & (0.1009) & $(0.0011)$ \\
\hline \multirow[t]{2}{*}{$\operatorname{Reg}$} & -0.0005 & -0.0001 & $-0.0004 * *$ & $0.0004 * *$ \\
\hline & $(0.0053)$ & $(0.0002)$ & $(0.0002)$ & $(0.0002)$ \\
\hline \multirow[t]{2}{*}{$J r$} & $-0.4482 * *$ & $0.0201 * * *$ & $-0.0778 * * *$ & $0.0584 * * *$ \\
\hline & $(0.1739)$ & $(0.0070)$ & $(0.0253)$ & $(0.0130)$ \\
\hline \multirow[t]{2}{*}{ Fdi } & $-0.0272 * *$ & -0.0002 & 0.0007 & $0.0008^{*}$ \\
\hline & $(0.0116)$ & $(0.0005)$ & $(0.0006)$ & $(0.0004)$ \\
\hline \multirow[t]{2}{*}{ Gov } & $0.0206^{* * * *}$ & $-0.0003 *$ & -0.0001 & -0.0001 \\
\hline & $(0.0044)$ & $(0.0002)$ & $(0.0002)$ & $(0.0004)$ \\
\hline \multirow[t]{2}{*}{$R s$} & $0.8213 * * *$ & $0.0436^{* * *}$ & $0.0744 * * *$ & $0.0683 * * *$ \\
\hline & $(0.2732)$ & $(0.0110)$ & $(0.0131)$ & $(0.0077)$ \\
\hline \multirow[t]{2}{*}{ Constant } & $14.3676^{* * *}$ & $0.5536^{* * *}$ & $-0.9843 * * *$ & $-0.6889 * * *$ \\
\hline & $(2.7081)$ & $(0.1093)$ & $(0.3859)$ & $(0.3373)$ \\
\hline Observations & 1995 & 1995 & 1710 & 1710 \\
\hline$R$-squared & 0.091 & 0.153 & 0.153 & 0.153 \\
\hline C-D Wald-F & & & 130.198 & 130.198 \\
\hline
\end{tabular}

注: 括号内为稳健标准误, $* * * * * * *$ 分别表示在 $1 \% 、 5 \%$ 和 $10 \%$ 的水平上显著,下同。

表3 数字金融分维度对碳排放绩效的影响

Table 3 Impact of various dimensions of digital finance on carbon emission performance

\begin{tabular}{|c|c|c|c|c|c|c|}
\hline \multirow{2}{*}{ 变量 } & \multicolumn{2}{|c|}{ 数字金融覆盖度 } & \multicolumn{2}{|c|}{ 数字金融使用深度 } & \multicolumn{2}{|c|}{ 数字化程度 } \\
\hline & $\mathrm{Ci}$ & $\mathrm{Ce}$ & $\mathrm{Ci}$ & $\mathrm{Ce}$ & $\mathrm{Ci}$ & $\mathrm{Ce}$ \\
\hline \multirow[t]{2}{*}{ Bre } & $-0.0050 * * *$ & $0.0004 * * *$ & & & & \\
\hline & $(0.0013)$ & $(0.0001)$ & & & & \\
\hline \multirow[t]{2}{*}{ Dep } & & & -0.0001 & $0.0004 * * *$ & & \\
\hline & & & $(0.0009)$ & $(0.0000)$ & & \\
\hline \multirow[t]{2}{*}{ Dig } & & & & & $-0.0020 * * *$ & $0.0001^{* * *}$ \\
\hline & & & & & $(0.0007)$ & $(0.0000)$ \\
\hline \multirow[t]{2}{*}{ Constant } & $11.6999 * * *$ & $0.3821 * * *$ & $20.5174 * * *$ & $0.4711 * * *$ & $15.9865^{* * *}$ & -0.0242 \\
\hline & $(2.7252)$ & $(0.1113)$ & $(2.2836)$ & $(0.0911)$ & $(2.1296)$ & $(0.0877)$ \\
\hline 控制变量 & 是 & 是 & 是 & 是 & 是 & 是 \\
\hline 固定效应 & 是 & 是 & 是 & 是 & 是 & 是 \\
\hline Observations & 1995 & 1995 & 1995 & 1995 & 1995 & 1995 \\
\hline$R$-squared & 0.095 & 0.137 & 0.087 & 0.169 & 0.091 & 0.120 \\
\hline
\end{tabular}

理论分析表明数字金融发展通过经济增长效 应、产业结构效应和技术创新效应 3 种渠道提升了 碳排放绩效。本文基于式(2)和式 (3)对 3 种机制进
行检验。从表 4 的回归结果可以看出数字金融发展 显著促进了经济增长、产业结构升级和技术创新， 这与理论分析一致。 
表 4 数字金融对中介变量的影响

Table 4 Impact of digital finance on intermediary variables

\begin{tabular}{lccc}
\hline \multicolumn{1}{c}{ 变量 } & $R g d p$ & Ins & Tec \\
\hline$D f$ & $0.0148^{* * *}$ & $0.0018^{* * *}$ & $0.0086^{* * *}$ \\
& $(0.0009)$ & $(0.0001)$ & $(0.0004)$ \\
Constant & 3.0912 & $5.3962^{* * *}$ & 1.0611 \\
& $(2.1416)$ & $(0.1714)$ & $(0.9135)$ \\
控制变量 & 是 & 是 & 是 \\
固定效应 & 是 & 是 & 是 \\
Observations & 1995 & 1995 & 1995 \\
$R$-squared & 0.524 & 0.732 & 0.662 \\
\hline
\end{tabular}

需要注意的是,经济增长和碳排放可能存在反 向因果关系, 导致内生性问题。一方面, 随着经济 发展水平的提升, 居民和政府将会更加重视环境质 量,追求可持续发展,政府将增加污染治理投资,促 进碳减排; 另一方面,政府环境规制强度的增加可 能会增加企业生产成本,挤占企业研发投资,对企 业的生产率和竞争力产生消极影响,但也有可能倒 逼企业技术创新,通过“创新补偿”效应,促进经济 增长。上述反向因果问题可能导致回归结果的偏 误,因此本文采用动态面板系统 GMM模型估计经
济增长对碳排放绩效的影响,结果如表 5 第 1 和第 2 列所示, 最后几行给出了模型设定检验结果, 可以 看到 Sargan 检验的 $p$ 值大于 0.1 , 接受了工具变量有 效的原假设, $A R(2)$ 检验接受了误差项不存在二阶 序列相关的原假设, 因此 GMM 模型的估计结果是 有效的。

从表 5 第 $1 、 3 、 5$ 列的结果可以看出经济发展水 平 $(R g d p)$ 、产业结构 $(I n s)$ 和技术水平 $(T e c)$ 的系数 均为负且显著, 说明数字金融发展通过经济增长效 应、产业结构效应和技术创新效应对碳排放强度产 生了降低的效果。中介效应模型认为,核心解释变量 的系数不显著时,需要进行 Sobel检验以确定中介效 应是否显著,第 5 列的结果中数字金融指数 $(D f)$ 的系 数并不显著,需要进行 Sobel 检验, 检验结果表明 Sobel 统计量为 5.634 , 在 $1 \%$ 的水平上显著,这说明 存在以技术水平 $(\mathrm{Tec})$ 为中介变量的中介效应。

从表 5第 $2 、 4 、 6$ 列的结果可以看出经济发展水 平 $(R g d p)$ 和技术水平 $(T e c)$ 的系数均为正, 且在 $1 \%$ 的水平上显著, 说明数字金融发展通过经济增长效

表 5 中介变量对碳排放绩效的影响

Table 5 Impact of intermediary variables on carbon emission performance

\begin{tabular}{|c|c|c|c|c|c|c|}
\hline \multirow{2}{*}{ 变量 } & \multicolumn{2}{|c|}{ 经济增长效应 } & \multicolumn{2}{|c|}{ 产业结构效应 } & \multicolumn{2}{|c|}{ 技术创新效应 } \\
\hline & $\mathrm{Ci}$ & $\mathrm{Ce}$ & $\mathrm{Ci}$ & $\mathrm{Ce}$ & $\mathrm{Ci}$ & $\mathrm{Ce}$ \\
\hline \multirow[t]{2}{*}{$D f$} & $-0.0027 * * *$ & $0.0003 * * *$ & $-0.0064 * * *$ & $0.0005 * * *$ & 0.0003 & $0.0005 * * *$ \\
\hline & $(0.0006)$ & $(0.0001)$ & $(0.0013)$ & $(0.0001)$ & $(0.0013)$ & $(0.0001)$ \\
\hline \multirow[t]{2}{*}{$\operatorname{Rgdp}$} & $-0.0531 * * *$ & $0.0022 * * *$ & & & & \\
\hline & $(0.0146)$ & $(0.0006)$ & & & & \\
\hline \multirow[t]{2}{*}{ Ins } & & & $-1.7816^{* * *}$ & 0.0073 & & \\
\hline & & & $(0.3806)$ & $(0.0155)$ & & \\
\hline \multirow[t]{2}{*}{$\mathrm{Tec}$} & & & & & $-0.4102 * * *$ & $0.0096 * * *$ \\
\hline & & & & & $(0.0712)$ & $(0.0029)$ \\
\hline Constant & 0.0299 & $-0.1010 * * *$ & 4.7539 & $0.5931 * * *$ & $14.8028 * * *$ & $0.5637 * * *$ \\
\hline 控制变量 & 是 & 是 & 是 & 是 & 是 & 是 \\
\hline 固定效应 & 是 & 是 & 是 & 是 & 是 & 是 \\
\hline Observations & 1710 & 1710 & 1995 & 1995 & 1995 & 1995 \\
\hline$R$-squared & & & 0.102 & 0.153 & 0.108 & 0.158 \\
\hline$A R(1)$ & 0.0012 & 0.0211 & & & & \\
\hline$A R(2)$ & 0.2013 & 0.2315 & & & & \\
\hline Sargan & 0.3984 & 0.4051 & & & & \\
\hline
\end{tabular}

注: $A R$ 检验和 Sargen 检验均为 $p$ 值。 
大小存在异质性,在低经济发展水平和低金融发展

综上所述,数字金融发展通过经济增长效应、 产业结构效应和技术创新效应降低了碳排放强度， 通过经济增长效应和技术创新效应提升了碳排放 效率。总体来看, 数字金融发展可通过以上 3 种效 应改善碳排放绩效。

\section{5 异质性分析}

数字金融与传统金融服务相比服务成本和服 务门槛更低, 覆盖范围、使用深度和数字化程度更 深, 能够为落后和偏远地区的弱势群体提供金融服 务, 缓解金融排斥和金融抑制。为明确数字金融发 展对不同地区碳排放绩效的影响,需要进行异质性 分析。依据经济发展水平、传统金融发展水平的中 位数对总样本进行分类并采用双固定效应模型进 行回归,结果如表 6 和表 7 所示。

(1) 碳排放强度 $(C i)$ 视角。表 6 中数字金融指 数 $(D f)$ 的指数均为负, 说明在不同的分组中数字金 融发展均促进了碳排放强度的降低,但减排效应的 水平的城市减排效应更大。这些城市主要分布在 中部和西部地区, 存在较为严重的金融抑制和金融 排斥, 数字金融的重要性质之一即为普惠性,数字 金融降低了金融服务成本和门槛, 为弱势群体提供 了金融服务,促进了中部和西部地区的经济发展， 因此数字金融在这些地区内城市的碳减排效应 更高。

(2) 碳排放效率 $(C e)$ 视角。如表 7 所示,在不同 分组的回归结果均显示数字金融发展对碳排放效 率产生了正向影响,在经济发展水平和金融发展水 平高的地区,对碳排放效率的改善作用更大。这些 城市主要分布在经济发展水平较高的东部地区,其 本身的金融资源条件和工业基础都较好,数字化技 术的应用将大幅提高要素配置效率和能源利用效 率,提升碳排放效率。改进碳排放效率的充分必要 条件即为绿色技术创新,完备的基础设施和丰富的 人力资本为研发活动提供了良好的外部性条件,数 字金融的发展更易激发技术创新,对碳排放效率产

\section{表 6 数字金融发展对碳排放绩效影响的异质性: 碳排放强度}

Table 6 Heterogeneity analysis: carbon emission intensity

\begin{tabular}{|c|c|c|c|c|}
\hline \multirow{2}{*}{ 变量 } & \multicolumn{2}{|c|}{ 经济发展水平异质性 } & \multicolumn{2}{|c|}{ 金融发展水平异质性 } \\
\hline & 高经济发展水平 $C i$ & 低经济发展水平 $C i$ & 高金融发展水平 $C i$ & 低金融发展水平 $C i$ \\
\hline \multirow[t]{2}{*}{$D f$} & $-0.0031^{*}$ & $-0.0115 * * *$ & $-0.0025 * * *$ & $-0.0072 * * *$ \\
\hline & $(0.0019)$ & $(0.0016)$ & $(0.0007)$ & $(0.0028)$ \\
\hline \multirow[t]{2}{*}{ Constant } & $-8.9681^{*}$ & $16.6421 * * *$ & $3.6607 * *$ & $19.8369 * * *$ \\
\hline & (5.1019) & (3.1656) & $(1.8169)$ & (5.9704) \\
\hline 控制变量 & 是 & 是 & 是 & 是 \\
\hline 固定效应 & 是 & 是 & 是 & 是 \\
\hline Observations & 998 & 997 & 998 & 997 \\
\hline$R$-squared & 0.045 & 0.256 & 0.137 & 0.113 \\
\hline
\end{tabular}

表 7 数字金融发展对碳排放绩效影响的异质性: 碳排放效率

Table 7 Heterogeneity analysis: carbon emission efficiency

\begin{tabular}{|c|c|c|c|c|}
\hline \multirow{2}{*}{ 变量 } & \multicolumn{2}{|c|}{ 经济发展水平异质性 } & \multicolumn{2}{|c|}{ 金融发展水平异质性 } \\
\hline & 高经济发展水平 $\mathrm{Ce}$ & 低经济发展水平 $\mathrm{Ce}$ & 高金融发展水平 $C e$ & 低金融发展水平 $C e$ \\
\hline \multirow[t]{2}{*}{$D f$} & $0.0006 * * *$ & $0.0003^{* * *}$ & $0.0006 * * *$ & $0.0003 * * *$ \\
\hline & $(0.0001)$ & $(0.0000)$ & $(0.0001)$ & $(0.0001)$ \\
\hline \multirow[t]{2}{*}{ Constant } & $0.7860 * * *$ & $0.3402 * * *$ & $0.6405 * * *$ & $0.3275^{* * *}$ \\
\hline & $(0.2850)$ & $(0.0719)$ & $(0.2342)$ & $(0.1120)$ \\
\hline 控制变量 & 是 & 是 & 是 & 是 \\
\hline 固定效应 & 是 & 是 & 是 & 是 \\
\hline Observations & 998 & 997 & 998 & 997 \\
\hline$R$-squared & 0.147 & 0.180 & 0.192 & 0.137 \\
\hline
\end{tabular}


生提升的作用。

\section{6 稳健性分析}

为提高本文实证结论的可信度,进一步采用替 换核心解释变量、剔除直辖市的方法进行稳健性检 验, 具体如下。

(1)替换核心解释变量。数字金融发展水平与 数字经济发展密切相关,本文采用腾讯研究院公布 的数字经济指数 (2015-2018) 作为核心解释变量, 将样本数据缩减为 2015-2018 年的面板数据, 检验 数字金融发展对碳排放绩效的影响,结果如表 8 第 1 和第 2 列所示, 可以看到核心解释变量数字经济指 数 $(D e)$ 的系数显著为负和正, 说明数字金融发展降 低了碳排放强度,增加了碳排放效率, 改善了碳排 放绩效,说明前文的实证结果是稳健的。

(2)剔除直辖市。由于中国城市发展水平各 异, 尤其是直辖市北京、天津、上海、重庆的经济体 量远大于其他城市, 本文对这几个直辖市样本进行 剔除,再次检验数字金融发展对碳排放绩效的影 响,结果如表 8 第 3 和第 4 列所示, 可以看到核心解 释变量数字金融指数 $(D f)$ 的系数与前文的回归结 果并无显著差异,证实前文的实证结果是稳健的。

\section{5 结论与建议}

\section{1 结论}

在数字经济时代, 实现碳达峰、碳中和, 是一场 广泛而深刻的经济社会系统性变革, 需要利用数字 金融形成减污降碳的长效机制。本文以中国285个 城市 2012-2018年的平衡面板数据作为研究样本,
将碳排放绩效分为碳排放强度和碳排放效率, 并运 用不同的实证方法检验了数字金融发展的碳减排 效应与作用路径。主要结论如下:

(1)数字金融发展显著降低了城市碳排放强度 并提高了城市碳排放效率。中国的数字金融具备 环境友好型特征,数字化技术与金融服务的结合能 够通过减少现金使用、减少线下交易活动产生碳减 排效应,引导金融资源流向绿色低碳的环境友好型 企业,改善城市碳排放绩效。

(2)数字金融分维度回归结果表明数字金融覆 盖广度、使用深度、数字化程度的提升均改善了碳 排放绩效,表明数字技术在金融领域的应用打破了 传统金融服务在城市内部的区位限制，提高了金融 产品的丰富性和可得性,并促进了金融流通效率的 提升,有助于改善城市碳排放绩效。

（3）中介效应分析表明,数字金融发展通过经 济增长效应、产业结构效应和技术创新效应改善了 碳排放绩效。这说明金融服务的数字化转型有助 于激发城市经济增长潜能,便于为企业研发活动提 供资金支持,也能通过引导金融资源流向绿色低碳 行业促进产业结构优化, 从而改善城市碳排放 绩效。

(4)异质性分析结果表明: (1)数字金融发展更 有利于降低低经济发展水平和低金融发展水平的 城市的碳排放强度。这说明数字技术的应用促进 了金融服务在欠发达城市的覆盖, 促进了当地经济 的增长和产业结构的升级,产生了更强的碳减排效

表 8 各类稳健性检验结果

Table 8 Results of various robustness tests

\begin{tabular}{|c|c|c|c|c|}
\hline \multirow{2}{*}{ 变量 } & \multicolumn{2}{|c|}{ 缩减样本: 替换核心解释变量 } & \multicolumn{2}{|c|}{ 缩减样本:剔除直辖市 } \\
\hline & $\mathrm{Ci}$ & $\mathrm{Ce}$ & $\mathrm{Ci}$ & $\mathrm{Ce}$ \\
\hline \multirow[t]{2}{*}{$D e$} & $-0.0101 * *$ & $0.0137 * * *$ & & \\
\hline & $(0.0051)$ & $(0.0008)$ & & \\
\hline \multirow[t]{2}{*}{$D f$} & & & $-0.0033^{* * *}$ & $0.0004 * * *$ \\
\hline & & & $(0.0012)$ & $(0.0000)$ \\
\hline \multirow[t]{2}{*}{ Constant } & $15.3175 * * *$ & $-0.2980 * * *$ & $14.3709 * * *$ & $0.4859 * * *$ \\
\hline & $(2.3751)$ & $(0.1021)$ & $(2.7230)$ & $(0.0956)$ \\
\hline 控制变量 & 是 & 是 & 是 & 是 \\
\hline 固定效应 & 是 & 是 & 是 & 是 \\
\hline Observations & 1140 & 1140 & 1967 & 1967 \\
\hline$R$-squared & 0.079 & 0.386 & 0.190 & 0.158 \\
\hline
\end{tabular}


应。(2)数字金融发展更有利于提升高经济发展水 平和高金融发展水平的城市的碳排放效率。经济 较为发达的城市拥有的完备的基础设施和丰富的 人力资本, 为研发活动提供了良好的外部性条件, 更易发挥数字金融的技术创新效应。

\section{2 建议}

本文从碳排放绩效的新视角对数字金融发展 的影响进行了细致深人分析, 丰富了相关的理论体 系, 并对其中介效应和异质性进行分析, 可以为发 挥数字金融的碳减排效应提供针对性的实证依据 和政策启示:

(1)以数字金融促进低碳经济发展。数字金融 发展有效改善了碳排放绩效, 因此应继续大力发展 数字金融,完善数字基础设施建设, 促进金融服务 与现代数字技术结合, 提升各类金融产品的可得性 和可利用性, 为绿色转型活动提供多样化的金融产 品组合, 降低绿色转型面临的风险。发挥数字金融 环境友好优势, 运用数字技术完善金融资源配置机 制, 通过市场化环境工具解决碳排放的负外部性, 如数字金融与绿色金融的结合, 可以有效完善绿色 信贷的激励和约束机制, 有助于碳达峰和碳中和目 标的实现。

(2) 以数字金融服务引导绿色创新。借助数字 化平台优化资本配置,引导金融资源流向环境友好 的、具有创新潜力的能源企业,为能源技术的研发 提供充足资金支持, 提高能源开发和利用水平, 各 地区应抓住机遇,推进创新驱动发展战略,完善基 础设施建设和人才引进政策, 为绿色创新提供良好 的外部条件。

(3) 因地制宜, 充分发挥数字红利。在中西部 地区应继续承接东部地区的产业转移, 同时以数据 要素赋能传统资源要素, 激发经济增长潜能,发挥 规模经济红利, 提升各类资源要素的利用效率, 促 进经济可持续发展; 在东部地区应促进绿色创新和 产业结构优化, 发挥技术创新效应和产业结构效 应, 提升碳排放绩效, 助力碳达峰和碳中和。

\section{参考文献(References)：}

[1] 贺茂斌, 杨晓维. 数字普惠金融、碳排放与全要素生产率[J]. 金 融论坛, 2021, 26(2): 18-25. [He M B, Yang X W. Digital inclusive finance, carbon emissions and total factor productivity[J]. Financial Forum, 2021, 26(2): 18-25.]

[2]陈矜, 许一帆. 数字金融发展降低环境污染了吗?[J]. 辽宁科技 大学学报, 2020, 43(5): 69-74. [Chen J, Xu Y F. Does the development of digital finance reduce environmental pollution?[J]. Journal of University of Science and Technology Liaoning, 2020, 43 (5): 69-74.]

[3] 许到, 高显, 霍治方. 数字金融的污染减排效应[J]. 财经科学, 2021, (4): 28-39. [Xu Z, Gao Y, Huo Z F. Research on pollution effect of digital finance[J]. Finance \& Economics, 2021, (4): 2839.]

[ 4] Artur T, Chousa J P, Vadlamannati K C. Does higher economic and financial development lead to environmental degradation: Evidence from BRIC countries[J]. Energy Policy, 2009, 37(1): 246253.

[5] Ouyang Y F, Li P. On the nexus of financial development, economic growth, and energy consumption in China: New perspective from a GMM panel VAR approach[J]. Energy Economics, 2018, 71 (3): $238-252$.

[6] 贺俊, 程锐, 刘庭. 金融发展、技术仜新与环境污染[J]. 东北大学 学报(社会科学版), 2019, 21(2): 139-148. [He J, Cheng R, Liu T Financial development, technological innovation and environmental pollution[J]. Journal of Northeast University (Social Science Edition), 2019, 21(2): 139-148.]

[7] Sadorsky P. The impact of financial development on energy consumption in emerging economies[J]. Energy Policy, 2010, 38(5): 2528-2535.

[ 8 ] Sorrell S, Dimitropoulos J, Sommerville M. Empirical estimates of the direct rebound effect: A review[J]. Energy Policy, 2009, 37(4): 1356-1371.

[9]邵汉华,刘耀涁. 金融发展与碳排放的非线性关系研究: 基于面 板平滑转换模型的实证检验[J]. 软科学, 2017, 31(5): 80-84. [Shao H H, Liu Y B. The nonlinear relationship between financial development and carbon emissions: Based on panel smooth transition regression model[J]. Soft Science, 2017, 31(5): 80-84.]

[10] 彭智敏, 向念, 夏克郁. 长江经济带地级城市金融发展与碳排放 关系研究[J]. 湖北社会科学, 2018, (11): 32-38. [Peng Z M, Xiang N, Xia K Y. Research on the relationship between financial development and carbon emission in prefecture-level cities in the Yangtze River Economic Belt[J]. Hubei Social Sciences, 2018 (11): 32-38.]

[11] Grossman G M, Krueger A B. Environmental impacts of a North American free trade agreement[J]. CEPR Discussion Papers, 
1992, 8(2): 223-250.

[12] 严成樑, 李涛, 兰伟. 金融发展、创新与二氧化碳排放[J]. 金融研 究, 2016, (1): 14-30. [Yan C L, Li T, Lan W. Financial development, innovation and carbon emissions[J]. Journal of Financial Research, 2016, (1): 14-30.]

[13] 熊灵, 齐绍洲. 金融发展与中国省区碳排放: 基于 STIRPAT 模 型和动态面板数据分析[J]. 中国地质大学学报(社会科学版), 2016, 16(2): 63-73. [Xiong L, Qi S Z. Financial development and carbon emission in China: Based on STIRPAT model and dynamic panel data analysis[J]. Journal of China University of Geosciences (Social Sciences Edition), 2016, 16(2): 63-73.]

[14] 陈碧琼, 张梁梁. 动态空间视角下金融发展对碳排放的影响力 分析[J]. 软科学, 2014, 28(7): 140-144. [Chen B Q, Zhang L L. The impact of financial development on carbon emissions from the perspective of dynamic spatial[J]. Soft Science, 2014, 28(7): 140144.]

[15] 胡宗义, 李毅. 金融发展对环境污染的双重效应与门槛特征 [J]. 中国软科学, 2019, (7): 68-80. [Hu Z Y, Li Y. Dual effects and threshold characteristics of financial development on environmental pollution[J]. China Soft Science, 2019, (7): 68-80.]

[16] 滕否, 马德功. 数字金融能够促进高质量发展吗?[J]. 统计研究, 2020, 37(11): 80-92. [Teng L, Ma D G. Can digital finance help to promote high quality development[J]. Statistical Research, 2020, 37(11): 80-92.]

[17] 王永仓, 温涛. 数字金融的经济增长效应及异质性研究 [J]. 现代 经济探讨, 2020, (11): 56-69. [Wang Y C, Wen T. Research on economic growth effect and heterogeneity of digital finance[J]. Modern Economic Research, 2020, (11): 56-69.]

[18] 唐松, 伍旭川, 祝佳. 数字金融与企业技术创新: 结构特征、机制 识别与金融监管下的效应差异 [J]. 管理世界, 2020, 36(5): 5266. [Tang S, Wu X C, Zhu J. Digital finance and enterprise technology innovation: Structural feature, mechanism identification and effect difference under financial supervision[J]. Management World, 2020, 36(5): 52-66.]

[19] 杜金岷, 韦施威, 吴文洋. 数字普惠金融促进了产业结构优化 吗?[J]. 经济社会体制比较, 2020, (6): 38-49. [Du J M, Wei S W, Wu W Y. Does digital financial inclusive promote the optimization of industrial structure?[J]. Comparative Economic \& Social Systems, 2020, (6): 38-49.]

[20] 许广月, 宋德勇. 中国碳排放环境库兹涅茨曲线的实证研究: 基 于省域面板数据[J]. 中国工业经济, 2010, (5): 37-47. [Xu G Y, Song D Y. An empirical study of the environmental Kuznets Curve of China's carbon emission: Based on provincial panel data[J]. China Industrial Economy, 2010, (5): 37-47.]

[21] 赫永达, 文红, 孙传旺. “十四五” 期间我国碳排放总量及其结构 预测: 基于混频数据 ADL-MIDAS 模型[J]. 经济问题, 2021, (4):
31-40. [He Y D, Wen H, Sun C W. Forecasting China's total carbon emission and its structure in the 14th Five Year Plan: Based on Mixed-frequency ADL-MIDAS model[J]. On Economic Problems, 2021, (4): 31-40.]

[22]王鹏, 谢丽文. 污染治理投资、企业技术创新与污染治理效率 [J]. 中国人口・资源与环境, 2014, 24(9): 51-58. [Wang P, Xie L W. Pollution control investment, enterprise technological innovation and pollution control efficiency[J]. China Population, Resources and Environment, 2014, 24(9): 51-58.]

[23] 陈阳, 逯进, 于平. 技术创新减少环境污染了吗? 来自中国 285 个城市的经验证据[J]. 西安交通大学学报(社会科学版), 2019, 39(1): 73-84. [Chen Y, Lu J, Yu P. Does technology innovation reduce environmental pollution? Empirical research based on 285 Chinese cities[J]. Journal of Xi' an Jiaotong University (Social Sciences), 2019, 39(1): 73-84.]

[24] 陈超凡, 蓝庆新, 王泽. 城市创新行为改善生态效率了吗? 基于 空间关联与溢出视角的考察 [J]. 南方经济, 2021, (1): 102-119. [Chen C F, Lan Q X, Wang Z. Does urban innovation improve ecological efficiency? Based on the perspective of spatial correlation and spatial spillover[J]. Southern China Journal of Economics, 2021, (1): 102-119.]

[25] 刘玉珂, 金声甜. 中部六省能源消费碳排放时空演变特征及影 响因素 [J]. 经济地理, 2019, 39(1): 182-191. [Liu Y K, Jin S T. Temporal and spatial evolution characteristics and influencing factors of energy consumption carbon emissions in six provinces of central China[J]. Economic Geography, 2019, 39(1): 182-191.]

[26] 赵领娣, 张磊, 徐乐, 等. 人力资本、产业结构调整与绿色发展效 率的作用机制 [J]. 中国人口·资源与环境, 2016, 26(11): 106114. [Zhao L D, Zhang L, Xu L, et al. Mechanism of human capital, industrial structure adjustment and green development efficiency[J]. China Population, Resources and Environment, 2016, 26 (11): 106-114.]

[27] 钱海章, 陶云清, 曹松威, 等. 中国数字金融发展与经济增长的 理论与实证 [J]. 数量经济技术经济研究, 2020, 37(6): 26-46. [Qian H Z, Tao Y Q, Cao S W, et al. Theoretical and empirical analysis on the development of digital finance and economic growth in China[J]. The Journal of Quantitative \& Technical Economics, 2020, 37(6): 26-46.]

[28] 温忠麟, 张雷, 侯杰泰, 等. 中介效应检验程序及其应用[J]. 心理 学报, 2004, (5): 111-117. [Wen Z L, Zhang L, Hou J T, et al. Testing and application of the mediating effects[J]. Acta Psychologica Sinica, 2004, (5): 111-117.]

[29] 史丹, 李少林. 排污权交易制度与能源利用效率: 对地级及以上 城市的测度与实证[J]. 中国工业经济, 2020, (9): 5-23. [Shi D, Li S L. Emissions trading system and energy use efficiency: Measurements and empirical evidence for cities at and above the pre- 
fecture level[J]. China Industrial Economics, 2020, (9): 5-23.]

[30] 马大来, 陈仲常, 王玲. 中国省际碳排放效率的空间计量[J]. 中 国人口·资源与环境, 2015, 25(1): 67-77. [Ma D L, Chen Z C, Wang L. Spatial econometrics research on inter-provincial carbon emissions efficiency in China[J]. China Population, Resources and Environment, 2015, 25(1): 67-77.]

[31] 王少剑, 高爽, 黄永源, 等. 基于超效率 SBM 模型的中国城市碳 排放强度时空演变格局及预测 [J]. 地理学报, 2020, 75(6): 1316-1330. [Wang S J, Gao S, Huang Y Y, et al. Spatial-temporal evolution and trend prediction of urban carbon emission performance in China based on super- efficiency SBM model[J]. Acta Geographica Sinica, 2020, 75(6): 1316-1330.]

[32] Chen H X, Zhang X L, Wu R W, et al. Revisiting the environmental Kuznets curve for city-level $\mathrm{CO}_{2}$ emissions: Based on corrected NPP-VIIRS nighttime light data in China[J]. Journal of Cleaner Production, 2020, DOI: 10.1016/j.jclepro.2020.121575.

[33] Meng X, Han J, Huang C. An improved vegetation adjusted nighttime light urban index and its application in quantifying spatiotemporal dynamics of carbon emissions in China[J]. Remote Sensing, 2017, DOI: 10.3390/rs9080829.

[34] Zhang W S, Jiang L, Cui Y Z, et al. Effects of urbanization on airport $\mathrm{CO}_{2}$ emissions: A geographically weighted approach using nighttime light data in China[J]. Resources, Conservation and Recycling, 2019, DOI: 10.1016/j.resconrec.2019.104454.

[35] 吴健生, 牛妍, 彭建, 等. 基于 DMSP/OLS 夜间灯光数据的 19952009 年中国地级市能源消费动态 [J]. 地理研究, 2014, 33(4): 625-634. [Wu J S, Niu Y, Peng J, et al. Research on energy consumption dynamic among prefecture-level cities in China based on DMSP/OLS nighttime light[J]. Geographical Research, 2014, 33 (4): 625-634.]

[36] 郭峰, 王靖一, 王芳, 等. 测度中国数字普惠金融发展: 指数编制
与空间特征[J]. 经济学(季刊), 2020, 19(4): 1401-1418. [Guo F, Wang J Y, Wang F, et al. Measuring China's digital financial inclusion: Index compilation and spatial characteristics[J]. China Economic Quarterly, 2020, 19(4): 1401-1418.]

[37] 白俊红, 蒋伏心. 考虑环境因素的区域创新效率研究: 基于三阶 段 DEA 方法[J]. 财贸经济, 2011, (10): 104-112. [Bai J H, Jiang F X. Research on regional innovation efficiency considering environmental factors: Based on three-stage DEA method[J]. Finance \& Trade Economics, 2011, (10): 104-112.]

[38] 付凌晖. 我国产业结构高级化与经济增长关系的实证研究[J]. 统计研究, 2010, 27(8): 79-81. [Fu L H. Research on regional innovation efficiency with environment factors: Based on threestage DEA model[J]. Statistical Research, 2010, 27(8): 79-81.]

[39] 王少剑, 黄永源. 中国城市碳排放强度的空间溢出效应及驱动 因素[J]. 地理学报, 2019, 74(6): 1131-1148. [Wang S J, Huang Y Y. Spatial spillover effect and driving factors of carbon emission intensity at city level in China[J]. Acta Geographica Sinica, 2019, 74(6): 1131-1148.]

[40] 周迪, 罗东权. 绿色税收视角下产业结构变迁对中国碳排放的 影响 [J]. 资源科学, 2021, 43(4): 693-709. [Zhou D, Luo D Q. Green taxation, industrial structure transformation and carbon emissions reduction[J]. Resources Science, 2021, 43(4): 693-709.]

[41] 王霞, 张丽君, 秦耀辰, 等. 中国高碳制造业碳排放时空演变及 其驱动因素 [J]. 资源科学, 2020, 42(2): 323-333. [Wang X, Zhang L J, Qin Y C, et al. Spatiotemporal changes of carbon emissions in high-carbon manufacturing industry in China and driving factors[J]. Resources Science, 2020, 42(2): 323-333.]

[42] 广嫦娥, 路江林. 环境规制对绿色技术创新的影响研究: 来自湖 南省的证据[J]. 经济经纬, 2019, 36(2): 126-132. [Kuang C E, Lu J L. Research on the influence of environmental regulation on green technology innovation: Evidence from Hunan Province[J]. 
Economic Survey, 2019, 36(2): 126-132.]

[43] 易行健, 周利. 数字普惠金融发展是否显著影响了居民消费?

来自中国家庭的微观证据[J]. 金融研究, 2018, (11): 47-67. [Yi
X J, Zhou L. Does digital financial inclusion significantly influence household consumption? Evidence from household survey data in China[J]. Financial Research, 2018, (11): 47-67.]

\title{
The impact of urban digital finance development on carbon emission performance in China and mechanism
}

\author{
DENG Rongrong, ZHANG Aoxiang \\ (School of Economics Management and Law, University of South China, Hengyang 421001, China)
}

\begin{abstract}
Based on a theoretical analysis, this study used the balanced panel data of 285 city samples in China from 2012 to 2018 to empirically analyze the impact of digital finance development on carbon emission performance by using the panel regression model and instrumental variable method. The results show that: (1) The development of digital finance significantly reduces the carbon emission intensity and improves the carbon emission efficiency, and the improvement of the coverage, use depth, and digitalization degree of digital finance improves the carbon emission performance. (2) The analysis of intermediary mechanism shows that the development of digital finance reduces carbon emission intensity through economic growth effect, industrial structure effect, and technological innovation effect, and improves carbon emission efficiency through economic growth effect and technological innovation effect. In general, the development of digital finance improves carbon emission performance through these three effects. (3) The results of heterogeneity analysis show that the development of digital finance is more conducive to reducing the carbon emission intensity of cities with low economic development level and low financial development level; with regard to carbon emission efficiency, the development of digital finance is more conducive to improving the carbon emission efficiency of cities with high economic development level and high financial development level, which is a result of the different basic conditions of technological innovation.
\end{abstract}

Key words: digital finance; carbon emission intensity; carbon emission efficiency; intermediary mechanism; heterogeneity effect; China 